\title{
Compensatory changes in the lipid composition of the erythrocyte membrane
}

Circulating lipoproteins can influence the lipid composition of erythrocyte membranes and consequently membrane function. Owen and co-workers described this phenomenon in a recent TIBS article $^{1}$ and also speculated that erythrocytes are able to compensate for changes in lipid composition and membrane function. We would like to present experimental evidence which supports the speculations of Owen et al.

observed that an increased intake of cholesterol as the only dietary variable increased the C:PL ratio of erythrocytes. Table I shows that this effect was associated with a decrease and increase in the relative percentage of stearic acid (18:0) and linoleic acid (18:2) in erythrocyte phospholipids. In another trial $^{4}$, healthy human subjects added $15 \mathrm{ml}$ of cod-liver oil to their daily diet. It was found that a marked increase in

Table I. Lipid composition of erythrocytes from rabbits

\begin{tabular}{|c|c|c|c|}
\hline & \multirow[t]{2}{*}{ C:PL ratio } & \multicolumn{2}{|c|}{ PL fatty acids ( $\mathrm{mol} \%$ ) } \\
\hline & & 18:0 & $18: 2$ \\
\hline Controls $(n=7)$ & $1.21 \pm 0.16$ & $26.5 \pm 2.0$ & $5.5 \pm 4.4$ \\
\hline Cholesterol-fed $(n=6)$ & $1.40 \pm 0.05$ & $23.7 \pm 2.1$ & $9.1 \pm 3.4$ \\
\hline
\end{tabular}

Rabbits were fed diets with $(0.2 \%, \mathrm{w} / \mathrm{w})$ or no cholesterol for 43 days ${ }^{3}$.

Table II. Mean lipid composition of erythrocytes from patients with LCAT deficiency

\begin{tabular}{lccccc}
\hline & C:PL & SM:PC & \multicolumn{2}{c}{ PL fatty acids (mol \%) } \\
\cline { 5 - 6 } & ratio & ratio & $18: 2$ & $20: 4$ \\
\hline Healthy subjects $^{5}$ & 0.82 & 0.75 & 10.3 & 18.3 \\
Hereditary LCAT deficiency $^{5}$ & 0.91 & 0.26 & 15.7 & 13.5 \\
Secondary LCAT deficiency $^{6}$ & 1.05 & 0.54 & 10.4 & 14.4 \\
\hline
\end{tabular}

The lipid fluidity of the erythrocyte membrane affects several functional properties of the membrane such as flexibility, receptor activity and oxygen transport ${ }^{2}$. Determinants of lipid fluidity are the fatty acid composition of the lipids, the nature and ratios of the phospholipid classes and the cholesterol:phospholipid (C:PL) ratio. An increase in the degree of unsaturation of phospholipid (PL) fatty acids increases the lipid fluidity, whereas an increase in the sphingomyelin:phosphatidyl choline (SM:PC) ratio and in the C:PL ratio decreases the membrane lipid fluidity ${ }^{2}$. Several observations support the possibility that compensatory changes controlling homeostasis of the lipid fluidity can occur.

In an experiment ${ }^{3}$ with rabbits we the polyunsaturated $\omega-3$ fatty acid, eicosapentaenoic acid (20:5), was accompanied by a significant decrease in the polyunsaturated $\omega-6$ fatty acid, linoleic acid (18:2), in erythrocyte phosphatidylcholine. No change in the C:PL ratio of erythrocytes was observed. These experiments suggest that induction of a change in the lipid composition which tends to alter fluidity of the erythrocyte membrane is counteracted by a change with an opposite effect on fluidity.

Table II shows that in patients with hereditary plasma lecithin-cholesterol acyl transferase (LCAT) deficiency there is an increase in the erythrocyte C:PL ratio and a decrease in the proportion of arachidonic acid (20:4) in $\mathrm{PL}^{5}$. These changes in lipid composition are counteracted by changes which increase lipid fluidity, namely a decreased SM:PC ratio and an increased proportion of linoleic acid in phospholipids (Table II). In patients with LCAT deficiency secondary to liver disease, the increased erythrocyte $\mathrm{C}: \mathrm{PL}$ ratio and decreased content of $20: 4$ fatty acid in phospholipids are associated with a lowered SM:PC ratio, but the percentage of 18:2 fatty acid in phospholipids is not increased. Possibly liver disease restricts the ability of erythrocytes to compensate for changes in their lipid composition (see Ref. 1).

There is no doubt that the lipid fluidity of cell membranes can be altered by $\operatorname{diet}^{7}$ and by LCAT deficiency ${ }^{5,6}$. We have presented evidence however, that compensatory mechanisms are present to minimize changes in erythrocyte membrane fluidity and (probably as a result of this effect) to stabilize membrane function.

\section{References}

1 Owen, J. S., McIntyre, N. and Gillet, M. P. T. (1984) Trends Biochem. Sci. 9, 238-242

2 Cooper, R. A. (1977) N. Engl. J. Med. 297 , 371-377

3 Schouten, J. A., Beynen, A. C., Popp-Snijders, C. and Van der Veen, E. A. (1984) Nutr. Rep. Int. 29, 1223-1232

4 Popp-Snijders, C., Schouten, J. A., De Jong, A. P. and Van der Veen, E. A. (1984) Scand. J. Clin. Lab. Invest. 44, 39-46

5 Jain, S. K., Mohandas, N., Sensabaugh, G. F., Shojania, A. M. and Shohet, S. B. (1982) J. Lab. Clin. Med. 99, 816-826

6 Owen, J. S., Bruckdorfer, K. R., Day, R. C. and McIntyre, N. (1982) J. Lipid Res. 23, 124-132

7 Goodnight, S. H. Jr, Harris, W. S., Connor, W. E. and Illingworth, D. R. (1982) Arteriosclerosis 2, 87-113

A. C. BEYNEN

Department of Laboratory Animal Science, State University,

3508 TD Utrecht, The Netherlands.

$$
\begin{aligned}
& \text { J. A. SCHOUTEN } \\
& \text { C. POPP-SNIJDERS }
\end{aligned}
$$

Department of Internal Medicine,

Free University Hospital,

1007 MB Amsterdam, The Netherlands. 